NOTE

\title{
Novel dolphin morbillivirus (DMV) outbreak among Mediterranean striped dolphins Stenella coeruleoalba in Italian waters
}

\author{
Alessandra Pautasso" ${ }^{1}$, Barbara Iulini ${ }^{1}$, Carla Grattarola1 ${ }^{1}$, Federica Giorda $^{1,2}$, \\ Maria Goria ${ }^{1}$, Simone Peletto ${ }^{1}$, Loretta Masoero ${ }^{1}$, Walter Mignone ${ }^{1}$, Katia Varello ${ }^{1}$, \\ Antonio Petrella ${ }^{3}$, Antonio Carbone ${ }^{3}$, Antonio Pintore ${ }^{4}$, Daniele Denurra ${ }^{4}$, \\ Francesco Scholl ${ }^{5}$, Antonella Cersini ${ }^{5}$, Roberto Puleio ${ }^{6}$, Giuseppa Purpari ${ }^{6}$, \\ Giuseppe Lucifora ${ }^{7}$, Giovanna Fusco ${ }^{7}$, Giovanni Di Guardo ${ }^{8}$, Sandro Mazzariol ${ }^{9}$, \\ Cristina Casalone ${ }^{1, *}$ \\ ${ }^{1}$ Istituto Zooprofilattico Sperimentale del Piemonte Liguria e Valle d'Aosta, 10154 Torino, Italy \\ ${ }^{2}$ Institute for Animal Health and Food Safety (IUSA), Faculty of Veterinary Medicine, University of Las Palmas de Gran Canaria, \\ 35413 Arucas, Las Palmas, Canary Islands, Spain \\ ${ }^{3}$ Istituto Zooprofilattico Sperimentale della Puglia e Basilicata, 71121 Foggia, Italy \\ ${ }^{4}$ Istituto Zooprofilattico Sperimentale della Sardegna, 07100 Sassari, Italy \\ ${ }^{5}$ Istituto Zooprofilattico Sperimentale del Lazio e della Toscana, 00178 Roma, Italy \\ ${ }^{6}$ Istituto Zooprofilattico Sperimentale della Sicilia, 90129 Palermo, Italy \\ ${ }^{7}$ Istituto Zooprofilattico Sperimentale del Mezzogiorno, 80055 Portici, Italy \\ ${ }^{8}$ Faculty of Veterinary Medicine, University of Teramo, 64100 Teramo, Italy \\ ${ }^{9}$ University of Padova, Department of Comparative Biomedicine and Food Science (BCA), 35020 Legnaro-Padova, Italy
}

\begin{abstract}
An unusual mortality event (UME) of striped dolphins Stenella coeruleoalba occurred in the period July to December 2016 along the Italian Ionian coastline. We conducted a complete postmortem examination on 28 specimens and detected dolphin morbillivirus (DMV), by means of biomolecular analyses, in the target tissues of 17 animals. Unlike previous outbreaks occurring in the Mediterranean Sea in 2011 and 2013, we observed typical pathological changes suggestive of morbilliviral infection in an acute/subacute phase and immunohistochemical reactivity. The same findings were observed in 13 other specimens beached along the Italian coastline during 2016 with no temporal and geographical relationship with the ongoing epidemic outbreak. Molecular characterization and phylogenetic analysis showed that DMV sequences detected in Italy in 2016 clustered with those identified in Portugal and Galicia (Spain), representing a novel DMV strain of Atlantic origin which entered the Mediterranean Sea and affected a naïve striped dolphin population. DMV sequences detected in the previous Mediterranean outbreaks exhibited a marked genetic relatedness and diverged from those detected in cetaceans stranded along the Galician and Portuguese coasts since 2007.
\end{abstract}

KEY WORDS: Morbillivirus · Unusual mortality event $\cdot$ Striped dolphins $\cdot$ Italian Sea $\cdot$ RT-PCR

\section{INTRODUCTION}

Dolphin morbillivirus (DMV) is considered a pathogen of concern for free-ranging cetaceans, having been responsible for outbreaks of lethal disease world- wide (Van Bressem et al. 2014). In the Mediterranean Sea, 2 well documented DMV outbreaks, involving mainly striped dolphins Stenella coeruleoalba, occurred in 1990-1992 and 2006-2008 (Domingo et al. 1992, Raga et al. 2008). 
A decline in DMV immunity was reported in striped dolphins in the Western Mediterranean basin during the inter-epidemic period (Van Bressem et al. 2001, Bento et al. 2016), suggesting that limited viral circulation and waning protective immunity may increase the vulnerability of this species to sporadic and recurrent epidemics. Both outbreaks started near the Strait of Gibraltar, suggesting the introduction of the virus to an immunologically naïve population, from DMV-infected cetaceans of Atlantic origin (Van Bressem et al. 2014). In recent years, unusual mortality events (UMEs) associated with DMV infection were reported in Spain and Italy (2011-2013) (Rubio Guerri et al. 2013, Casalone et al. 2014).

Viral strains responsible for Mediterranean outbreaks exhibited a marked genetic relatedness, while the epidemiology of the infection changed depending on many factors, such as the herd immunity and the density of the susceptible population (Casalone et al. 2014, Van Bressem et al. 2014). Indeed, in contrast with the 1990-1992 and the 2006-2008 epidemics, during the last UMEs that occurred in Spain and Italy (2011-2013), despite high levels of biomolecular positivity to DMV, the infected animals showed milder lesions and pathological changes not directly attributable to DMV but rather suggestive of a chronic systemic DMV infection in immunosuppressed animals with secondary infections (Van Bressem et al. 2014). The morbillivirus antigen was seldom revealed by immunostaining in target tissues. Mainly juvenile individuals were affected (Rubio Guerri et al. 2013, Casalone et al. 2014), probably due to a lack of specific antiviral immunity (Casalone et al. 2014).

Furthermore, an expansion in the host range of this virus in the Mediterranean basin, recently detected in 'new' hosts such as fin whales Balaenoptera physalus (Mazzariol et al. 2016) and Cuvier's beaked whales Ziphius cavirostris (Centelleghe et al. 2017), is of great concern and suggests the role of other species as potential reservoirs for viral maintenance and persistence in the marine environment. All of these observations argue in favor of an endemic DMV circulation in the Western Mediterranean and the likely occurrence of cyclic outbreaks when the herd immunity decreases (Rubio Guerri et al. 2013).

Genetically, DMV circulating in the Mediterranean showed a close relationship with viruses circulating in the adjacent waters of the Central East Atlantic Ocean (Canary Islands) (Bellière et al. 2011b, Sierra et al. 2014, Beffagna et al. 2017). Biological and genetic studies support the hypothesis of contacts occurring through the Strait of Gibraltar between populations of striped dolphins of the Western Mediterranean Sea and Central East Atlantic Ocean favoring inter-animal transmission of viruses (Sierra et al. 2014). In contrast, phylogenetic analysis of DMV sequences from cetaceans stranded along Galician and Portuguese coasts (NE Atlantic) since 2007 showed that these sequences diverged from those reported in the Mediterranean and Canary Islands (Bento et al. 2016). These observations enforced previous data (Valsecchi et al. 2004, Bourret et al. 2007) that support the relative isolation between cetacean populations of the Atlantic coast of Portugal and northern Spain and those of the Canary Islands and the Mediterranean (Bento et al. 2016).

Here, we report the first cetacean UME in the Mediterranean Sea caused by a DMV strain genetically related to that recently characterized from dolphins stranded along the Portuguese and Galician coasts and clearly different from the DMV strains thus far detected in the Mediterranean Sea.

During 2016, the Italian Diagnostic Report on Stranded Cetaceans reported 256 stranded cetaceans along Italian coastlines. In the period July-December 2016, a marked rise in the number of cetacean strandings, i.e. 5 times the $10 \mathrm{yr}$ average recorded for the same time period, was documented along the Ionian coast of Italy. Fifty-seven cetaceans were involved, including 51 striped dolphins, 1 bottlenose dolphin Tursiops truncatus and 5 odontocetes of undetermined species.

\section{MATERIALS AND METHODS}

\subsection{Samples}

A complete postmortem examination was performed according to standardized protocols (Geraci \& Lounsbury 2005) on 28 striped dolphin carcasses stranded during July-December 2016 along the Ionian coast of Italy (Apulia, Basilicata, Calabria and Sicily). Age classes were estimated from total body length, since direct techniques (e.g. counting growth layers in teeth) were not applied systematically due to logistic difficulties (Mazzariol et al. 2015).

Target tissue samples were collected and, based on the degree of postmortem decomposition, stored frozen at $-80^{\circ} \mathrm{C}$ for microbiological and biomolecular investigations to identify the main cetacean pathogens (DMV, Verna et al. 2017; Brucella sp., OIE 2015; Toxoplasma gondii, Vitale et al. 2013; herpesvirus, VanDevanter et al. 1996). Tissues were also preserved in $10 \%$ neutral-buffered formalin for histo- 
pathology and immunohistochemistry (IHC). The same protocol was applied to 42 additional carcasses (Stenella coeruleoalba, $\mathrm{n}=27$; Tursiops truncatus, $\mathrm{n}=$ 9; Risso's dolphins Grampus griseus, $\mathrm{n}=3$; sperm whales Physeter macrocephalus, $\mathrm{n}=2$; and 1 shortbeaked common dolphin Delphinus delphis) beached in 2016 along the entire Italian coastline with no temporal relationship with the ongoing epidemic outbreak. The extreme degree of decomposition precluded such a diagnostic approach on 30 additional necropsied carcasses in 2016.

\subsection{DMV investigations: $P C R$, histology, IHC, isolation, sequence analysis}

DMV was detected by molecular techniques on tissue samples of major organs including the central nervous system (CNS), lung, spleen, lymph nodes, liver, kidney, heart, bladder, skin and muscle according to the availability of tissues, using different reverse transcription (RT)-PCR methods and confirmed by a new RT-PCR with degenerate primers targeting a $287 \mathrm{bp}$ fragment of the $\mathrm{N}$ gene (Verna et al. 2017). When the degree of postmortem decomposition allowed, tissues were processed by conventional histological techniques and subsequently embedded in paraffin, sectioned at $4 \pm 2 \mu \mathrm{m}$ and stained with hematoxylin and eosin (H\&E).

IHC for morbillivirus was performed on brain, lung and/or lymphoid tissues (including spleen and lymph nodes), according to the availability of tissues (Uchida et al. 1999). Appropriate positive and negative controls were included in the analysis. Viral isolation was attempted from a selection of PCR-positive frozen tissues, using the Vero.DogSLAMtag cell line (Nielsen et al. 2008, Peletto et al. 2018).

DMV infection was confirmed by sequencing RTPCR products. Molecular characterization and phylogenetic analysis were carried out using the protocols described by Bellière et al. (2011a) to investigate the evolutionary relationship of the detected strains. Maximum likelihood phylogenetic analysis was performed using MEGA 7.0 software on an $\mathrm{N}$ gene sequence dataset (176 bp) of DMV isolates, belonging to samples both from the Ionian coastline and the rest of Italy.

\section{RESULTS}

DMV was detected by molecular techniques in 17 out of 28 analyzed striped dolphins stranded along
Ionian coast of Italy during July-December 2016, in samples from CNS $(\mathrm{n}=15)$, lung $(\mathrm{n}=16)$, kidney $(\mathrm{n}=$ $13)$, spleen $(n=11)$, liver $(n=10)$, heart $(n=8)$, lymph nodes $(\mathrm{n}=9)$, bladder $(\mathrm{n}=2)$, skin $(\mathrm{n}=2)$ and muscle $(\mathrm{n}=2)$. All infected animals showed positivity in more than 1 tissue. In 15 cases, we observed positivity both in the CNS and lungs. Most DMV-infected striped dolphins were classified as 'newborn' (6/17) or 'juveniles' (7/17) and showed minimal postmortem decomposition (fresh carcasses or in moderate decomposition). Histological and IHC investigations were performed on 12 out of 17 infected striped dolphins.

Microscopically, typical pathological changes suggestive of morbilliviral infection in an acute/subacute phase were observed in 7/12 cases. The main findings observed were mild to moderate non-suppurative meningo-encephalitis with white matter vacuolization, syncytia, gliosis and neuronophagia along with broncho/bronchiolo-interstitial pneumonia with syncytia and lymphoid cell depletion. Furthermore, IHC analysis showed morbilliviral antigen in 6/12 animals, specifically in the brain $(n=5)$, lungs $(n=2)$, spleen $(\mathrm{n}=1)$ and lymph nodes $(\mathrm{n}=1)$. In the brain, positive multifocal staining was observed mainly in neurons, but also in glial cells and rarely in endothelial and meningeal cells (Fig. 1a). In the lungs, multifocal immunopositivity was detected in bronchial, bronchiolar and alveolar epithelia, in histiocytes and in syncytia; in the lymph nodes and spleen, morbilliviral antigens were demonstrated multifocally in macrophages and lymphoid cells. No other relevant coinfections were detected.

In addition to the animals involved in the Ionian outbreak, DMV was detected in 13 specimens (Stenella coeruleoalba, $\mathrm{n}=12$ and Delphinus delphis, $\mathrm{n}=1$ ) out of 42 animals beached and tested for DMV in 2016 along the entire Italian coastline without any temporal relationship to the ongoing epidemic outbreak. Microscopic and IHC findings were similar to those observed along the Ionian coastline (Italian Diagnostic Report on Stranded Cetaceans of 2016; www.salute.gov.it/imgs/C_17_pubblicazioni_2597_ allegato.pdf). Virus isolation attempts failed and no cytopathic effect was observed.

Phylogenetic analysis performed on 17 DMV isolates, belonging to samples both from the Ionian coastline (6/17) and the rest of Italy (11/17), showed that all DMV sequences cluster with those recently identified in Portugal and Galicia (Bento et al. 2016) (Fig. 2) and are separated from the Western Mediterranean and Canarian sequences. The same topology was confirmed by phylogenetic trees that were con- 

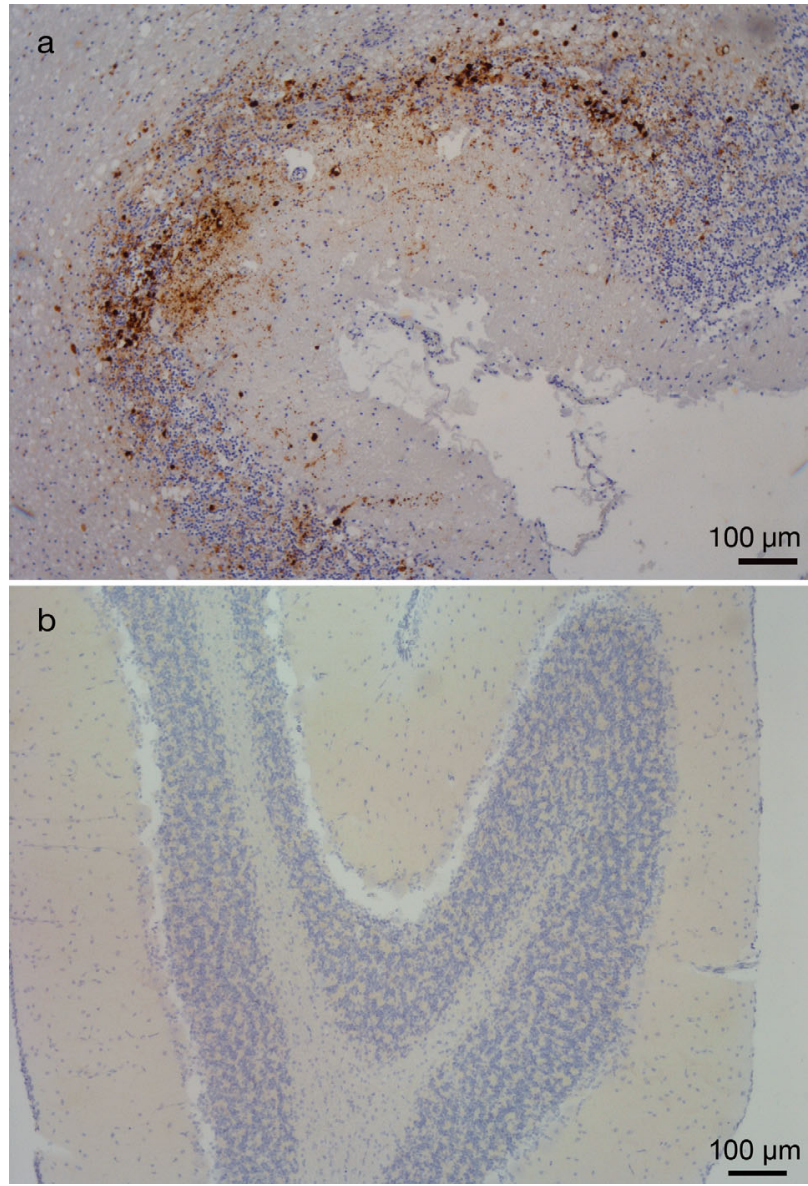

Fig. 1. Morbillivirus infection in the cerebellum of Stenella coeruleoalba. (a) Positive immunohistochemical labeling of morbilliviral antigen in granular and molecular layers, as well as in glial cells (dark staining). (b) Immunohistochemical examination of cerebellum of Stenella coeruleoalba.

Negative control of morbillivirus

structed on $\mathrm{P}(220 \mathrm{bp}), \mathrm{F}(429 \mathrm{bp})$ and $\mathrm{H}$ (295 bp) genes. Sequences $>200$ bp were submitted to GenBank (accession numbers MF472872-MF 472891).

\section{DISCUSSION}

Since 2016, we have been observing the introduction of a novel DMV strain along the Italian coastlines, which is similar to that recently identified in Portugal and Galicia (Bento et al. 2016) and was able to cause a new epidemic outbreak along the Ionian coast of Italy. Further investigations allowed us to confirm the circulation of the same strain along the entire Italian coast with no temporal or geographical relationship with the ongoing epidemic outbreak.

The pathological findings observed were suggestive of an acute/subacute disease that had not been observed associated with DMV epidemics in the Mediterranean basin since 2008 (Di Guardo \& Mazzariol 2016). Morbilliviruses are extremely virulent in immunologically naïve individuals, particularly juveniles lacking natural immunity. The introduction of new variants in such susceptible populations could allow for rapid transmission and epidemics.

Based on the biomolecular prevalence data, preliminary serological investigations and in the absence of mass mortality events, Bento et al. (2016) suggested an endemic circulation of this viral strain along NE Atlantic coasts. Our results indicate that

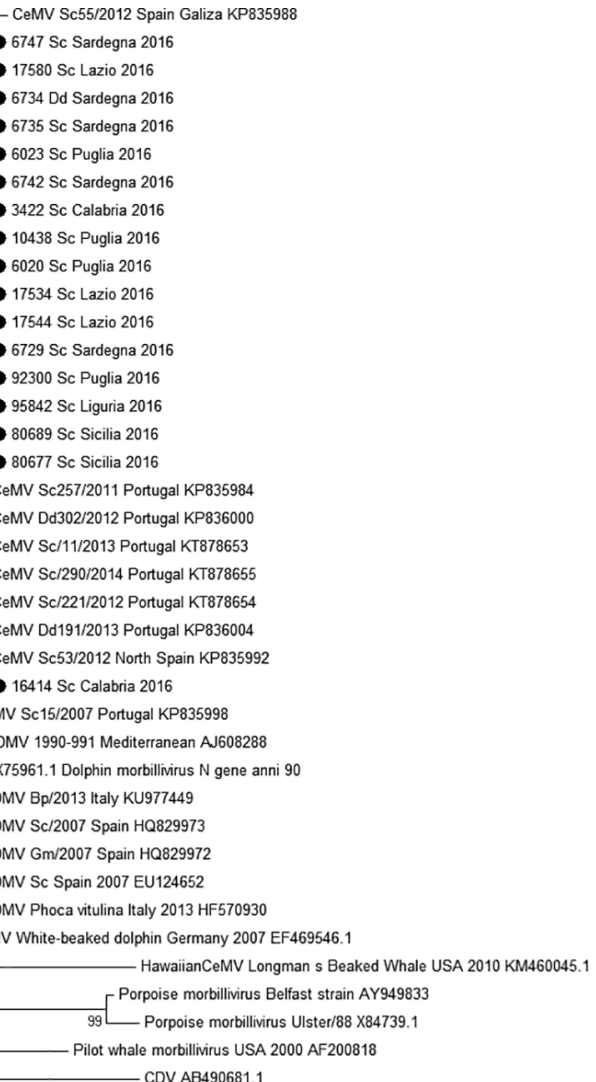

Fig. 2. Dolphin morbillivirus (DMV) phylogenetic tree constructed by maximum likelihood analysis (MEGA 7.0) from a $176 \mathrm{nt}$ alignment of the partial $\mathrm{N}$ gene. The tree includes 17 Italian isolates (identified by large dots) and sequences available in GenBank. Host species ( $\mathrm{Sc}=$ Stenella coeruleoalba; $\mathrm{Dd}=$ Delphinus delphis; $\mathrm{Gm}=$ Globicephala melas; $\mathrm{Bp}=$ Balaenoptera physalus), collection year and accession numbers are indicated for each sequence. Canine distemper virus (CDV) was used as the outgroup. Bootstrap values (1000 iterations) $>50$ are shown at the internal nodes. The branch length represents the sequence distance. The scale bar represents the percentage of nucleotide differences 
this DMV strain, presumably new to the Mediterranean basin, most likely entered immunologically naïve striped dolphins, resulting in a UME.

Our results also suggest that the striped dolphin population in the Western Mediterranean Sea/ Canary Islands is not as isolated from those in the NE Atlantic as previously thought (Bento et al. 2016). We suggest that NE Atlantic cetacean populations infected with the 'Portuguese-Galician strain' could have transmitted the infection to Mediterranean striped dolphins through occasional contacts in the Strait of Gibraltar.

Only a few cases were reported indicating the possible circulation of viruses between the NE Atlantic and the Mediterranean/Canary Islands. Specifically, phylogenetic analysis based on P gene sequences indicates that the 'Galician Portuguese strain' had been previously detected outside of the NE Atlantic area in 2 striped dolphins stranded, respectively, in the Canary Islands in 2011 (GenBank Accession number KJ139454) (Sierra et al. 2014) and in the Mediterranean in 2012 (KC572861). Similarly, Bento et al. (2016) reported 1 animal stranded in Portugal in 2007 infected with a DMV strain whose sequences (KP835995) clustered with samples from the Mediterranean/Canary Islands. More recently, a systemic infection produced by a DMV with a supposed similar NE Atlantic origin was described in a common dolphin stranded in Spain near Valencia on June 2016, indicating a likely transmission of the virus by contacts between NE Atlantic and Mediterranean populations of dolphins (Rubio Guerri et al. 2017).

In conclusion, our observations support an active circulation of a DMV strain of NE Atlantic origin along the Italian coastlines, most likely since 2016, diverging from those circulating in the Western Mediterranean and Canary Islands (Bellière et al. 2011b, Sierra et al. 2014, Beffagna et al. 2017). The high density and gregarious behavior of striped dolphins (Reeves et al. 2008) in the Mediterranean might be responsible for the propagation of the virus in Italian waters, and a similar scenario could not be excluded in other areas of the Mediterranean Sea.

Our next steps will be aimed at better understanding the epidemiologic and pathogenetic features of the infection caused by this DMV strain, including a full genome comparison with viral isolates recovered from previous outbreaks, as well as unraveling the role of other factors potentially modulating the susceptibility to infection, such as host genotype and immunological and environmental factors.
Acknowledgements. We thank the Italian Ministries of Health and Environment who supported the organization of the Italian Stranding network and funded this study under research projects.

\section{LITERATURE CITED}

Beffagna G, Centelleghe C, Franzo G, Di Guardo G, Mazzariol S (2017) Genomic and structural investigation on dolphin morbillivirus (DMV) in Mediterranean fin whales (Balaenoptera physalus). Sci Rep 7:41554

Bellière EN, Esperón F, Fernández A, Arbelo M, Muñoz MJ, Sánchez-Vizcaíno JM (2011a) Phylogenetic analysis of a new Cetacean morbillivirus from a short-finned pilot whale stranded in the Canary Islands. Res Vet Sci 90: 324-328

Bellière EN, Esperón F, Sánchez-Vizcaíno JM (2011b) Genetic comparison among dolphin morbillivirus in the 1990-1992 and 2006-2008 Mediterranean outbreaks. Infect Genet Evol 11:1913-1920

* Bento MC, Eira CI, Vingada JV, Marçalo AL and others (2016) New insight into dolphin morbillivirus phylogeny and epidemiology in the northeast Atlantic: opportunistic study in cetaceans stranded along the Portuguese and Galician coasts. BMC Vet Res 12:176

* Bourret VJR, Macé MRJM, Crouau-Roy B (2007) Genetic variation and population structure of western Mediterranean and northern Atlantic Stenella coeruleoalba populations inferred from microsatellite data. J Mar Biol Assoc UK 87:265-269

Casalone C, Mazzariol S, Pautasso A, Di Guardo G and others (2014) Cetacean strandings in Italy: an unusual mortality event along the Tyrrhenian Sea coast in 2013. Dis Aquat Org 109:81-86

Centelleghe C, Beffagna G, Palmisano G, Franzo G and others (2017) Dolphin morbillivirus in a Cuvier's beaked whale (Ziphius cavirostris), Italy. Front Microbiol 8:111

*Di Guardo G, Mazzariol S (2016) Cetacean morbillivirusassociated pathology: knowns and unknowns. Front Microbiol 7:112

* Domingo M, Visa J, Pumarola M, Marco AJ, Ferrer L, Rabanal R, Kennedy S (1992) Pathologic and immunocytochemical studies of morbillivirus infection in striped dolphins (Stenella coeruleoalba). Vet Pathol 29:1-10

Geraci JR, Lounsbury VJ (2005) Marine mammals ashore: a field guide for strandings, 2nd edn. National Aquarium in Baltimore, Baltimore, MD

Mazzariol S, Cozzi B, Centelleghe C (2015) Handbook for cetaceans' strandings. www.netcet.eu/files/Handbooks/ NETCET_Textbooks_on_veterinarian_operation_of_ceta ceans.pdf

Mazzariol S, Centelleghe C, Beffagna G, Povinelli M and others (2016) Mediterranean fin whales (Balaenoptera physalus) threatened by dolphin morbillivirus. Emerg Infect Dis 22:302-305

Nielsen O, Smith G, Weingartl H, Lair S, Measures L (2008) Use of a SLAM transfected Vero cell line to isolate and characterize marine mammal morbilliviruses using an experimental ferret model. J Wildl Dis 44:600-611

OIE (World Organisation for Animal Health) (2015) Manual of diagnostic tests and vaccines for terrestrial animals. OIE, Paris

Peletto S, Caruso C, Cerutti F, Modesto P and others (2018) Efficient isolation on Vero.DogSLAMtag cells and full 
genome characterization of dolphin morbillivirus (DMV) by next generation sequencing. Sci Rep 8:860

Raga JA, Banyard A, Domingo M, Corteyn M and others (2008) Dolphin morbillivirus epizootic resurgence, Mediterranean Sea. Emerg Infect Dis 14:471-473

Reeves RR, Stewart BS, Clapham PJ, Powell JA (2008) Guide to marine mammals of the world. National Audubon Society, New York, NY

Rubio-Guerri C, Melero M, Esperón F, Bellière EN and others (2013) Unusual striped dolphin mass mortality episode related to cetacean morbillivirus in the Spanish Mediterranean sea. BMC Vet Res 9:106

Rubio-Guerri C, Melero M, Crespo-Picazo JL, Jimenez MA, and others (2017) First case of dolphin morbillivirus in short-beaked common dolphin (Delphinus delphis) in Mediterranean Sea. 45th Symposium of the European Association for Aquatic Mammals, EAAM, Genova, p 26

Sierra E, Sánchez S, Saliki JT, Blas-Machado U, Arbelo M, Zucca D, Fernández A (2014) Retrospective study of etiologic agents associated with nonsuppurative meningoencephalitis in stranded cetaceans in the Canary Islands. J Clin Microbiol 52:2390-2397

Uchida K, Muranaka M, Horii Y, Murakami N, Yamaguchi R, Tateyama S (1999) Non-purulent meningoencephalomyelitis of a Pacific striped dolphin (Lagenorhynchus

Editorial responsibility: Stephen Raverty,

Abbotsford, British Columbia, Canada obliquidens). The first evidence of morbillivirus infection in a dolphin at the Pacific Ocean around Japan. J Vet Med Sci 61:159-162

* Valsecchi E, Amos W, Raga JA, Podestà M, Sherwin W (2004) The effects of inbreeding on mortality during a morbillivirus outbreak in the Mediterranean striped dolphin (Stenella coeruleoalba). Anim Conserv 7: 139-146

*Van Bressem M, Waerebeek KV, Jepson PD, Raga JA and others (2001) An insight into the epidemiology of dolphin morbillivirus worldwide. Vet Microbiol 81:287-304

* Van Bressem MF, Duignan PJ, Banyard A, Barbieri M and others (2014) Cetacean morbillivirus: current knowledge and future directions. Viruses 6:5145-5181

* VanDevanter DR, Warrener P, Bennet L, Schultz ER, Coulter S, Garber RL, Rose TM (1996) Detection and analysis of diverse herpesviral species by consensus primer PCR. J Clin Microbiol 34:1666-1671

Verna F, Giorda F, Miceli I, Rizzo G and others (2017) Detection of morbillivirus infection by RT-PCR RFLP analysis in cetaceans and carnivores. J Virol Methods 247:22-27

* Vitale M, Galluzzo P, Currò V, Gozdzik K, Schillaci D, Di Marco Lo Presti V (2013) A high sensitive nested PCR for Toxoplasma gondii detection in animal and food samples. J Microb Biochem Technol 5:39-41

Submitted: January 4, 2018; Accepted: November 20, 2018

Proofs received from author(s): January 16, 2019 